\title{
INTEGRAL REPRESENTATIONS FOR MULTIVARIATE LOGARITHMIC POLYNOMIALS
}

\author{
FENG QI \\ Institute of Mathematics, Henan Polytechnic University, Jiaozuo City, 454010, \\ Henan Province, China, Department of Mathematics, College of Science, Tianjin \\ Polytechnic University, Tianjin City, 300387, China
}

\begin{abstract}
In the paper, by induction and recursively, the author proves that the generating function of multivariate logarithmic polynomials and its reciprocal are a Bernstein function and a completely monotonic function respectively, establishes a Lévy-Khintchine representation for the generating function of multivariate logarithmic polynomials, deduces an integral representation for multivariate logarithmic polynomials, presents an integral representation for the reciprocal of the generating function of multivariate logarithmic polynomials, computes real and imaginary parts for the generating function of multivariate logarithmic polynomials, derives two integral formulas, and denies the uniform convergence of a known integral representation for Bernstein functions.
\end{abstract}

\section{CONTENTs}

1. Preliminaries and motivations 1

1.1. Completely monotonic function and the Bernstein function 2

1.2. Integral representations for the logarithmic function and its reciprocal 2

$\begin{array}{lll}\text { 1.3. Multivariate logarithmic polynomials } & 3\end{array}$

$\begin{array}{lll}2 . & \text { Integral representations for multivariate logarithmic polynomials } & 4\end{array}$

References

11

\section{Preliminaries and motivations}

In this section, we recall some preliminaries and state motivations of this paper.

E-mail address: qifeng618@gmail.com, qifeng618@hotmail.com

2010 Mathematics Subject Classification. Primary 11B83; Secondary 05A15, 11B37, 16A09, 26A48, 30A99, 33B99, 40A10, 44A10.

Key words and phrases. multivariate logarithmic polynomial; generating function; completely monotonic function; Bernstein function; integral representation; Lévy-Khintchine representation; real part; imaginary part; uniform convergence; recurrence relation; mathematical induction.

This paper was typeset using $\mathcal{A} \mathcal{M} \mathcal{S}$-LATEX. 
1.1. Completely monotonic function and the Bernstein function. Recall from [9, Chapter XIII], [13, Chapter 1], and [14, Chapter IV] that an infinitely differentiable function $f$ is said to be completely monotonic on an interval $I$ if it satisfies $(-1)^{k} f^{(k)}(x) \geq 0$ on $I$ for all $k \geq 0$. Theorem $12 \mathrm{~b}$ in [14] reads that a necessary and sufficient condition that $f(t)$ should be completely monotonic for $0<t<\infty$ is that

$$
f(t)=\int_{0}^{\infty} e^{-t s} \mathrm{~d} \alpha(s)
$$

where $\alpha(s)$ is non-decreasing and the integral converges for $0<s<\infty$.

Recall also from [13, p. 21, Definition 3.1] that a nonnegative function $f$ : $(0, \infty) \rightarrow \mathbb{R}$ is a Bernstein function if its first derivative $f^{\prime}$ is completely monotonic on $(0, \infty)$. Theorem 3.2 in [13] states that, a function $f:(0, \infty) \rightarrow[0, \infty)$ is a Bernstein function if and only if it admits the Lévy-Khintchine representation

$$
f(x)=a+b x+\int_{0}^{\infty}\left(1-e^{-x t}\right) \mathrm{d} \mu(t),
$$

where

$$
a=\lim _{t \rightarrow 0^{+}} f(t), \quad b=\lim _{t \rightarrow \infty} \frac{f(t)}{t},
$$

and $\mu$ is called the Lévy measure on $(0, \infty)$ satisfying $\int_{0}^{\infty} \min \{1, t\} \mathrm{d} \mu(t)<\infty$.

1.2. Integral representations for the logarithmic function and its reciprocal. We can directly verify by definition that $\ln (1+t)$ is a Bernstein function.

In [1, p. 230, 5.1.32] and [12, Remark 1], it is listed that

$$
\ln \frac{b}{a}=\int_{0}^{\infty} \frac{e^{-a u}-e^{-b u}}{u} \mathrm{~d} u .
$$

Letting $a=1$ and $b=1+t$ in 1.3 leads to

$$
\ln (1+t)=\int_{0}^{\infty} \frac{e^{-u}-e^{-(1+t) u}}{u} \mathrm{~d} u=\int_{0}^{\infty}\left(1-e^{-t u}\right) \frac{e^{-u}}{u} \mathrm{~d} u
$$

Comparing the integral representation (1.4), which is the Lévy-Khintchine representation of $\ln (1+t)$, with 1.2 shows that $\ln (1+t)$ is a Bernstein function.

In [2, Eq. (1.4)], [3, p. 2130], and [12, Lemma 1], it was proved that

$$
\frac{1}{\ln (1+z)}=\frac{1}{z}+\int_{1}^{\infty} \frac{1}{[\ln (t-1)]^{2}+\pi^{2}} \frac{\mathrm{d} t}{z+t}
$$

for $z \in \mathbb{C} \backslash(-\infty, 0]$. Then it is clear that $\frac{1}{\ln (1+t)}$ is a completely monotonic function on $(0, \infty)$. In [12, p. 996], it was presented that

$$
\frac{1}{\ln (1+t)}=\int_{0}^{\infty}\left[e^{-s} \int_{0}^{\infty} \frac{s^{u-1}}{\Gamma(u)} \mathrm{d} u\right] e^{-t s} \mathrm{~d} s,
$$

where $t>0$ and $\Gamma(z)$ is the classical gamma function which can be defined by

$$
\Gamma(z)=\int_{0}^{\infty} t^{z-1} e^{-t} \mathrm{~d} t, \quad \Re(z)>0 .
$$

Then it is easy to see that $\frac{1}{\ln (1+t)}$ is a completely monotonic function on $(0, \infty)$. Comparing 1.5 with 1.1 reveals that $\frac{1}{\ln (1+t)}$ is completely monotonic on $(0, \infty)$. This can also follows from the fact that $\ln (1+t)$ is a Bernstein function and the fact 
INTEGRAL REPRESENTATIONS OF MULTIVARIATE LOGARITHMIC POLYNOMIALS 3

in [4. pp. 161-162, Theorem 3] and [13, p. 64, Proposition 5.25] that the reciprocal of a Bernstein function is completely monotonic on $(0, \infty)$, but not conversely.

Proposition 3.6 in [13, p. 25] states that every Bernstein function $f$ has an extension $f: \overrightarrow{\mathbb{H}}=\{z \in \mathbb{C}: \Re(z) \geq 0\} \rightarrow \overrightarrow{\overrightarrow{\mathbb{H}}}$ which is continuous for $\Re(z) \geq 0$ and holomorphic for $\Re(z)>0$, satisfies $\overline{f(z)}=f(\bar{z})$ for all $z \in \overrightarrow{\mathbb{H}}=\{z \in \mathbb{C}: \Re(z)>0\}$, and has the representation

$$
f(z)=a+b z+\frac{2}{\pi} \int_{0}^{\infty} \frac{z}{z^{2}+s^{2}} \Re(f(s i)) \mathrm{d} s, \quad \Re(z) \geq 0,
$$

where $i=\sqrt{-1}$ is the imaginary unit, $a=\lim _{t \rightarrow 0^{+}} f(t)$, and $b=\lim _{t \rightarrow \infty} \frac{f(t)}{t}$. Theorem 3.1 in [5, 6, 7] reads that, if $\phi$ is a Bernstein function, then

$$
\phi(x)=b x+\frac{2}{\pi} \int_{0}^{\infty} \frac{x}{x^{2}+u^{2}} \Re[\phi(u i)] \mathrm{d} u .
$$

By virtue of (1.6) and (1.7), we see that the complex function $\ln (1+z)$ for $z \in$ $\mathbb{C} \backslash(-\infty,-1]$ has the integral representation

$$
\ln (1+z)=\frac{z}{\pi} \int_{0}^{\infty} \frac{\ln \left(1+s^{2}\right)}{z^{2}+s^{2}} \mathrm{~d} s, \quad \Re(z) \geq 0 ;
$$

The formula 1.8 can also be derived from taking $a=b=g=1$ and $c=z$ in

$$
\int_{0}^{\infty} \ln \left(a^{2}+b^{2} x^{2}\right) \frac{\mathrm{d} x}{c^{2}+g^{2} x^{2}}=\frac{\pi}{c g} \ln \frac{a g+b c}{g}, \quad a, b, c, g>0
$$

listed in [8, p. 564, Item 7].

1.3. Multivariate logarithmic polynomials. In [10, the notion "multivariate logarithmic polynomials" was introduced.

Definition 1.1 ([10, Definition 1.1]). For $x_{k} \in \mathbb{R}$ and $1 \leq k \leq m$, denote $\boldsymbol{x}_{m}=$ $\left(x_{1}, x_{2}, \ldots, x_{m-1}, x_{m}\right)$. Let $h(t)=\ln (1+t)$ for $t>-1$. Define $H\left(t ; \boldsymbol{x}_{m}\right)$ and $L_{m, n}\left(\boldsymbol{x}_{m}\right)$ by

$$
H\left(t ; \boldsymbol{x}_{m}\right)=h\left(x_{1} h\left(x_{2} h\left(\cdots x_{m-1} h\left(x_{m} h(t)\right) \cdots\right)\right)\right)=\sum_{n=1}^{\infty} L_{m, n}\left(\boldsymbol{x}_{m}\right) \frac{t^{n}}{n !} .
$$

We call $L_{m, n}\left(\boldsymbol{x}_{m}\right)$ higher order logarithmic polynomials, logarithmic polynomials of order $m, m$-variate logarithmic polynomials, multivariate logarithmic polynomials, logarithmic polynomials of $m$ variables $x_{1}, x_{2}, \ldots, x_{m}$, multi-order logarithmic polynomials alternatively. When $x_{1}=x_{2}=\cdots=x_{m-1}=x_{m}=1$, we denote $L_{m, n}(1, \ldots, 1)$ by $L_{m, n}$ and call them higher logarithmic numbers, logarithmic numbers of order $m$, and multi-order logarithmic numbers alternatively.

In the paper [10, the author established an explicit formula, an identity, and two recurrence relations for multivariate logarithmic polynomials $L_{m, n}\left(\boldsymbol{x}_{m}\right)$ by virtue of the Faà di Bruno formula and two identities of the Bell polynomials of the second kind in terms of the Stirling numbers of the first and second kinds and constructed some determinantal inequalities, product inequalities, logarithmic convexity for multivariate logarithmic polynomials $L_{m, n}\left(\boldsymbol{x}_{m}\right)$ by virtue of some properties of completely monotonic functions.

Naturally we pose a question: does the generating function $H\left(t ; \boldsymbol{x}_{m}\right)$ have similar properties to the above ones for $\ln (1+t)$ and its reciprocal? can these properties for 
$H\left(t ; \boldsymbol{x}_{m}\right)$ be applied to derive corresponding properties of multivariate logarithmic polynomials $L_{m, n}\left(\boldsymbol{x}_{m}\right)$ ?

\section{INTEGRAL REPRESENTATIONS FOR MULTIVARIATE LOGARITHMIC POLYNOMIALS}

In this section, when $x_{1}, x_{2}, \ldots, x_{m}>0$ and $m \in \mathbb{N}$, by induction and recursively, we prove that the generating function $H\left(t ; \boldsymbol{x}_{m}\right)$ and its reciprocal $\frac{1}{H\left(t ; \boldsymbol{x}_{m}\right)}$ are a Bernstein function and a completely monotonic function on $(0, \infty)$ respectively, establish a Lévy-Khintchine representation for $H\left(t ; \boldsymbol{x}_{m}\right)$, deduce an integral representation for multivariate logarithmic polynomials $L_{m, n}\left(\boldsymbol{x}_{m}\right)$, present an integral representation for $\frac{1}{H\left(t ; \boldsymbol{x}_{m}\right)}$, compute real and imaginary parts $\Re\left[H\left(t ; \boldsymbol{x}_{m}\right)\right]$ and $\Im\left[H\left(t ; \boldsymbol{x}_{m}\right)\right]$, derive two integral formulas, and deny the uniform convergence of the integral representations 1.6 and 1.7 .

Theorem 2.1. For $x_{1}, x_{2}, \ldots, x_{m}>0$, the generating function $H\left(t ; \boldsymbol{x}_{m}\right)$ is a Bernstein function and has the Lévy-Khintchine representation

$$
H\left(t ; \boldsymbol{x}_{m}\right)=\int_{0}^{\infty}\left(1-e^{-s_{m} t}\right) \int_{0}^{\infty} \cdots \int_{0}^{\infty} \mathcal{Q}\left(\boldsymbol{s}_{m} ; \boldsymbol{x}_{m}\right) \mathrm{d} s_{0} \cdots \mathrm{d} s_{m-1} \mathrm{~d} s_{m},
$$

where

$$
\mathcal{Q}\left(\boldsymbol{s}_{m} ; \boldsymbol{x}_{m}\right)=\left[\prod_{k=1}^{m} \frac{s_{k}^{s_{k-1} x_{k}}}{\Gamma\left(s_{k-1} x_{k}\right)}\right]\left[\prod_{\ell=0}^{m} \frac{e^{-s_{\ell}}}{s_{\ell}}\right]
$$

for $\boldsymbol{s}_{m}=\left(s_{0}, s_{1}, \ldots, s_{m}\right)$. Consequently, the multivariate logarithmic polynomials $L_{m, n}\left(\boldsymbol{x}_{m}\right)$ for $m, n \in \mathbb{N}$ can be represented by the integral

$$
L_{m, n}\left(\boldsymbol{x}_{m}\right)=(-1)^{n-1} \int_{0}^{\infty} \cdots \int_{0}^{\infty} s_{m}^{n} \mathcal{Q}\left(\boldsymbol{s}_{m} ; \boldsymbol{x}_{m}\right) \mathrm{d} s_{0} \cdots \mathrm{d} s_{m} .
$$

Proof. Item (iii) in [13, p. 28, Corollary 3.8] reads that the composition of two Bernstein functions is still a Bernstein function. Hence, it is immediate that the generating function $H\left(t ; \boldsymbol{x}_{m}\right)$ is a Bernstein function.

By virtue of (1.4), we have

$$
\begin{aligned}
H\left(t ; x_{1}\right) & =\int_{0}^{\infty}\left[1-e^{-u x_{1} \ln (1+t)}\right] \frac{e^{-u}}{u} \mathrm{~d} u \\
& =\int_{0}^{\infty}\left[1-\frac{1}{(1+t)^{u x_{1}}}\right] \frac{e^{-u}}{u} \mathrm{~d} u \\
& =\int_{0}^{\infty}\left[1-\frac{1}{\Gamma\left(u x_{1}\right)} \int_{0}^{\infty} v^{u x_{1}-1} e^{-v(1+t)} \mathrm{d} v\right] \frac{e^{-u}}{u} \mathrm{~d} u \\
& =\int_{0}^{\infty} \frac{1}{\Gamma\left(u x_{1}\right)}\left[\int_{0}^{\infty} v^{u x_{1}-1} e^{-v}\left(1-e^{-v t}\right) \mathrm{d} v\right] \frac{e^{-u}}{u} \mathrm{~d} u \\
& =\int_{0}^{\infty}\left(1-e^{-v t}\right) \int_{0}^{\infty} \frac{e^{-(u+v)}}{u} \frac{v^{u x_{1}-1}}{\Gamma\left(u x_{1}\right)} \mathrm{d} u \mathrm{~d} v
\end{aligned}
$$

Furthermore, it follows that

$$
\begin{aligned}
H\left(t ; \boldsymbol{x}_{2}\right) & =\int_{0}^{\infty}\left[1-e^{-v x_{2} \ln (1+t)}\right]\left[\int_{0}^{\infty} \frac{e^{-u}}{u} \frac{v^{u x_{1}-1}}{\Gamma\left(u x_{1}\right)} \mathrm{d} u\right] e^{-v} \mathrm{~d} v \\
& =\int_{0}^{\infty}\left[1-\frac{1}{(1+t)^{v x_{2}}}\right]\left[\int_{0}^{\infty} \frac{e^{-u}}{u} \frac{v^{u x_{1}-1}}{\Gamma\left(u x_{1}\right)} \mathrm{d} u\right] e^{-v} \mathrm{~d} v
\end{aligned}
$$


INTEGRAL REPRESENTATIONS OF MULTIVARIATE LOGARITHMIC POLYNOMIALS 5

$$
\begin{aligned}
& =\int_{0}^{\infty}\left[1-\frac{1}{\Gamma\left(v x_{2}\right)} \int_{0}^{\infty} w^{v x_{2}-1} e^{-w(1+t)} \mathrm{d} w\right]\left[\int_{0}^{\infty} \frac{e^{-u}}{u} \frac{v^{u x_{1}-1}}{\Gamma\left(u x_{1}\right)} \mathrm{d} u\right] e^{-v} \mathrm{~d} v \\
& =\int_{0}^{\infty} \frac{1}{\Gamma\left(v x_{2}\right)} \int_{0}^{\infty} w^{v x_{2}-1} e^{-w}\left(1-e^{-w t}\right) \mathrm{d} w\left[\int_{0}^{\infty} \frac{e^{-u}}{u} \frac{v^{u x_{1}-1}}{\Gamma\left(u x_{1}\right)} \mathrm{d} u\right] e^{-v} \mathrm{~d} v \\
& =\int_{0}^{\infty}\left(1-e^{-w t}\right) \int_{0}^{\infty} \int_{0}^{\infty} \frac{e^{-(u+v+w)}}{u} \frac{v^{u x_{1}-1}}{\Gamma\left(u x_{1}\right)} \frac{w^{v x_{2}-1}}{\Gamma\left(v x_{2}\right)} \mathrm{d} u \mathrm{~d} v \mathrm{~d} w
\end{aligned}
$$

and, inductively, that

$$
\begin{array}{r}
H\left(t ; \boldsymbol{x}_{m}\right)=\int_{0}^{\infty}\left(1-e^{-s_{m} t}\right) \int_{0}^{\infty} \cdots \int_{0}^{\infty} \frac{1}{s_{0}} \exp \left(-\sum_{\ell=0}^{m} s_{\ell}\right) \\
\times \prod_{k=1}^{m} \frac{s_{k}^{s_{k-1} x_{k}-1}}{\Gamma\left(s_{k-1} x_{k}\right)} \mathrm{d} s_{0} \cdots \mathrm{d} s_{m-1} \mathrm{~d} s_{m}
\end{array}
$$

which can be rearranged as 2.1 and again confirm that the generating function $H\left(t ; \boldsymbol{x}_{m}\right)$ is a Bernstein function.

Considering (1.9) in Definition 1.1, differentiating on both sides of 2.1 with respect to $t$, and taking the limit $t \rightarrow 0^{+}$derive 2.3 readily. The proof of Theorem 2.1 is complete.

Theorem 2.2. For $x_{1}, x_{2}, \ldots, x_{m}>0$, the function $\frac{1}{H\left(t ; \boldsymbol{x}_{m}\right)}$ is completely monotonic with respect to $t \in(0, \infty)$ and has the integral representation

$$
\frac{1}{H\left(t ; \boldsymbol{x}_{m}\right)}=\int_{0}^{\infty}\left[\int_{0}^{\infty} \cdots \int_{0}^{\infty} Q\left(\boldsymbol{s}_{m+1} ; \boldsymbol{x}_{m}\right) \mathrm{d} s_{0} \cdots \mathrm{d} s_{m}\right] e^{-s_{m+1} t} \mathrm{~d} s_{m+1},
$$

where

$$
Q\left(\boldsymbol{s}_{m+1} ; \boldsymbol{x}_{m}\right)=\left[\prod_{k=0}^{m} \frac{s_{k+1}^{s_{k} x_{k}}}{\Gamma\left(s_{k} x_{k}\right)}\right]\left[\prod_{\ell=1}^{m+1} \frac{e^{-s_{\ell}}}{s_{\ell}}\right]
$$

for $\boldsymbol{s}_{m+1}=\left(s_{0}, s_{1}, \ldots, s_{m+1}\right)$ and $x_{0}=1$.

Proof. Theorem 3.7 in [13, p. 27] states that $f$ is a Bernstein function if and only if $g \circ f$ is completely monotonic for every completely monotonic function $g$. Therefore, by induction, for $x_{1}, \ldots, x_{m}>0$, the function $\frac{1}{H\left(t ; \boldsymbol{x}_{m}\right)}$ is completely monotonic with respect to $t \in(0, \infty)$.

On the other hand, making use of 1.5 , we obtain

$$
\begin{aligned}
\frac{1}{H\left(t ; x_{1}\right)} & =\int_{0}^{\infty}\left[e^{-s} \int_{0}^{\infty} \frac{s^{u-1}}{\Gamma(u)} \mathrm{d} u\right] e^{-s x_{1} \ln (1+t)} \mathrm{d} s \\
& =\int_{0}^{\infty}\left[e^{-s} \int_{0}^{\infty} \frac{s^{u-1}}{\Gamma(u)} \mathrm{d} u\right] \frac{1}{(1+t)^{s x_{1}}} \mathrm{~d} s \\
& =\int_{0}^{\infty}\left[e^{-s} \int_{0}^{\infty} \frac{s^{u-1}}{\Gamma(u)} \mathrm{d} u\right] \frac{1}{\Gamma\left(s x_{1}\right)} \int_{0}^{\infty} v^{s x_{1}-1} e^{-v} e^{-v t} \mathrm{~d} v \mathrm{~d} s \\
& =\int_{0}^{\infty}\left[\int_{0}^{\infty} \int_{0}^{\infty} \frac{s^{u-1}}{\Gamma(u)} \frac{v^{s x_{1}-1}}{\Gamma\left(s x_{1}\right)} e^{-(s+v)} \mathrm{d} u \mathrm{~d} s\right] e^{-v t} \mathrm{~d} v
\end{aligned}
$$

Furthermore, it follows that 


$$
\begin{aligned}
& \frac{1}{H\left(t ; \boldsymbol{x}_{2}\right)}=\int_{0}^{\infty}\left[\int_{0}^{\infty} \int_{0}^{\infty} \frac{s^{u-1}}{\Gamma(u)} \frac{v^{s x_{1}-1}}{\Gamma\left(s x_{1}\right)} e^{-(s+v)} \mathrm{d} u \mathrm{~d} s\right] e^{-v x_{2} \ln (1+t)} \mathrm{d} v \\
& =\int_{0}^{\infty}\left[\int_{0}^{\infty} \int_{0}^{\infty} \frac{s^{u-1}}{\Gamma(u)} \frac{v^{s x_{1}-1}}{\Gamma\left(s x_{1}\right)} e^{-(s+v)} \mathrm{d} u \mathrm{~d} s\right] \frac{1}{(1+t)^{v x_{2}}} \mathrm{~d} v \\
= & \int_{0}^{\infty}\left[\int_{0}^{\infty} \int_{0}^{\infty} \frac{s^{u-1}}{\Gamma(u)} \frac{v^{s x_{1}-1}}{\Gamma\left(s x_{1}\right)} e^{-(s+v)} \mathrm{d} u \mathrm{~d} s\right] \frac{1}{\Gamma\left(v x_{2}\right)} \int_{0}^{\infty} w^{v x_{2}-1} e^{-w(1+t)} \mathrm{d} w \mathrm{~d} v \\
= & \int_{0}^{\infty}\left[\int_{0}^{\infty} \int_{0}^{\infty} \int_{0}^{\infty} \frac{s^{u-1}}{\Gamma(u)} \frac{v^{s x_{1}-1}}{\Gamma\left(s x_{1}\right)} \frac{w^{v x_{2}-1}}{\Gamma\left(v x_{2}\right)} e^{-(s+v+w)} \mathrm{d} u \mathrm{~d} s \mathrm{~d} v\right] e^{-w t} \mathrm{~d} w
\end{aligned}
$$

and, by induction, that

$$
\begin{aligned}
\frac{1}{H\left(t ; \boldsymbol{x}_{m}\right)}=\int_{0}^{\infty}\left[\int_{0}^{\infty} \cdots \int_{0}^{\infty}\right. & \int_{0}^{\infty} \prod_{k=0}^{m} \frac{s_{k}^{s_{k-1} x_{k}-1}}{\Gamma\left(s_{k-1} x_{k}\right)} \\
& \left.\times \exp \left(-\sum_{\ell=0}^{m} s_{\ell}\right) \mathrm{d} s_{-1} \mathrm{~d} s_{0} \cdots \mathrm{d} s_{m-1}\right] e^{-s_{m} t} \mathrm{~d} s_{m}
\end{aligned}
$$

which can be rewritten as 2.4 and confirm the complete monotonicity of $\frac{1}{H\left(t ; \boldsymbol{x}_{m}\right)}$ again. The proof of Theorem 2.2 is complete.

Remark 2.1. A straightforward computation gives

$$
\Re[H(s i ; x)]=\frac{1}{2} \ln \left[\left(1+\frac{1}{2} x \ln \left(1+s^{2}\right)\right)^{2}+x^{2} \arctan ^{2} s\right], \quad s, x>0 .
$$

Employing 1.6 results in

$$
\begin{aligned}
& H(t ; x)=\ln [1+x \ln (1+t)]=\frac{2}{\pi} \int_{0}^{\infty} \frac{t}{t^{2}+s^{2}} \Re(H(s i ; x)) \mathrm{d} s \\
& =\frac{1}{\pi} \int_{0}^{\infty} \frac{t}{t^{2}+s^{2}} \ln \left[\left(1+\frac{1}{2} x \ln \left(1+s^{2}\right)\right)^{2}+x^{2} \arctan ^{2} s\right] \mathrm{d} s
\end{aligned}
$$

for $t, x>0$. Letting $m=1$ in 2.1 or basing on its proof leads to

$$
H(t ; x)=\ln [1+x \ln (1+t)]=\int_{0}^{\infty}\left(1-e^{-v t}\right) \int_{0}^{\infty} \frac{v^{u x_{1}}}{\Gamma\left(u x_{1}\right)} \frac{e^{-(u+v)}}{u v} \mathrm{~d} u \mathrm{~d} v
$$

for $t, x>0$. Taking $m=1$ in 2.4 or retrospecting its proof reduces to

$$
\frac{1}{H(t ; x)}=\frac{1}{\ln [1+x \ln (1+t)]}=\int_{0}^{\infty} \int_{0}^{\infty} \int_{0}^{\infty} \frac{s^{u} v^{s x}}{\Gamma(u) \Gamma(s x)} \frac{e^{-(s+v)}}{s v} e^{-v t} \mathrm{~d} u \mathrm{~d} s \mathrm{~d} v
$$

for $t, x>0$.

We observe that these three integrals between (2.5) and (2.7) are respectively single, double, and triple and that the integrand in 2.5 is elementary but the integrands in 2.6 and 2.7 are not elementary. Consequently, we naturally pose a question: can one find out general integral representations, which are single integrals and whose integrands are elementary, for the generating function $H\left(t ; \boldsymbol{x}_{m}\right)$ and multivariate logarithmic polynomials $L_{m, n}\left(\boldsymbol{x}_{m}\right)$ ? One way to answer this question is to explicitly and elementarily express $\Re\left[H\left(s i ; \boldsymbol{x}_{m}\right)\right]$ and to apply the formula $(1.6)$. 
INTEGRAL REPRESENTATIONS OF MULTIVARIATE LOGARITHMIC POLYNOMIALS 7

Theorem 2.3. For $m \in \mathbb{N}$ and $s, x_{1}, \ldots, x_{m}>0$, the real and imaginary parts of the complex function $H\left(s i ; \boldsymbol{x}_{m}\right)$ can be recursively computed by

$$
\Re\left[H\left(s i ; \boldsymbol{x}_{m}\right)\right]=\frac{1}{2} \ln \left[U_{m}^{2}\left(x_{1}, \ldots, x_{m} ; s\right)+V_{m}^{2}\left(x_{1}, \ldots, x_{m} ; s\right)\right]
$$

and

$$
\Im\left[H\left(s i ; \boldsymbol{x}_{m}\right)\right]=\arctan \frac{V_{m}\left(x_{1}, \ldots, x_{m} ; s\right)}{U_{m}\left(x_{1}, \ldots, x_{m} ; s\right)},
$$

where $U_{m}\left(x_{1}, \ldots, x_{m} ; s\right)$ and $V_{m}\left(x_{1}, \ldots, x_{m} ; s\right)$ satisfy the recurrence relations

$$
U_{1}\left(x_{1} ; s\right)=1+\frac{1}{2} x_{1} \ln \left(s^{2}+1\right), \quad V_{1}\left(x_{1} ; s\right)=x_{1} \arctan s,
$$

and, when $m \geq 2$,

$$
\begin{gathered}
U_{m}\left(x_{1}, \ldots, x_{m} ; s\right)=1+\frac{1}{2} x_{1} \ln \left[U_{m-1}^{2}\left(x_{2}, \ldots, x_{m} ; s\right)+V_{m-1}^{2}\left(x_{2}, \ldots, x_{m} ; s\right)\right] \\
V_{m}\left(x_{1}, \ldots, x_{m} ; s\right)=x_{1} \arctan \frac{V_{m-1}\left(x_{2}, \ldots, x_{m} ; s\right)}{U_{m-1}\left(x_{2}, \ldots, x_{m} ; s\right)}
\end{gathered}
$$

Proof. By standard and careful calculation, we find

$$
\begin{aligned}
\Re\left[H\left(s i ; x_{1}\right)\right]= & \frac{1}{2} \ln \left\{\left[1+\frac{1}{2} x_{1} \ln \left(s^{2}+1\right)\right]^{2}+\left(x_{1} \arctan s\right)^{2}\right\} \\
\triangleq & \frac{1}{2} \ln \left[U_{1}^{2}\left(x_{1} ; s\right)+V_{1}^{2}\left(x_{1} ; s\right)\right] \\
\Im\left[H\left(s i ; x_{1}\right)\right]= & \arctan \frac{x_{1} \arctan s}{1+\frac{1}{2} x_{1} \ln \left(s^{2}+1\right)} \\
= & \arctan \frac{V_{1}\left(x_{1} ; s\right)}{U_{1}\left(x_{1} ; s\right)}, \\
\Re\left[H\left(s i ; \boldsymbol{x}_{2}\right)\right]= & \frac{1}{2} \ln \left\{\left[1+\frac{1}{2} x_{1} \ln \left\langle\left(1+\frac{1}{2} x_{2} \ln \left(s^{2}+1\right)\right)^{2}+\left(x_{2} \arctan s\right)^{2}\right\rangle\right]^{2}\right. \\
& \left.+\left[x_{1} \arctan \frac{x_{2} \arctan s}{1+\frac{1}{2} x_{2} \ln \left(s^{2}+1\right)}\right]^{2}\right\} \\
\triangleq & \frac{1}{2} \ln \left[U_{2}^{2}\left(x_{1}, x_{2} ; s\right)+V_{2}^{2}\left(x_{1}, x_{2} ; s\right)\right] \\
\Im\left[H\left(s i ; \boldsymbol{x}_{2}\right)\right]= & \arctan \frac{x_{1} \arctan \frac{x_{2} \arctan s}{1+\frac{1}{2} x_{2} \ln \left(1+s^{2}\right)}}{1+\frac{1}{2} x_{1} \ln \left(\left[1+\frac{1}{2} x_{2} \ln \left(1+s^{2}\right)\right]^{2}+\left[x_{2} \arctan s\right]^{2}\right)} \\
= & \arctan \frac{V_{2}\left(x_{1}, x_{2} ; s\right)}{U_{2}\left(x_{1}, x_{2} ; s\right)},
\end{aligned}
$$

and

$$
\begin{aligned}
\Re\left[H\left(s i ; \boldsymbol{x}_{3}\right)\right]= & \frac{1}{2} \ln \left\{\left[1+\frac{1}{2} x_{1} \ln \left\langle\left( 1+\frac{1}{2} x_{2} \ln \left(\left(1+\frac{1}{2} x_{3} \ln \left(s^{2}+1\right)\right)^{2}\right.\right.\right.\right.\right. \\
& \left.\left.\left.\left.+\left(x_{3} \arctan s\right)^{2}\right)\right)^{2}+\left(x_{2} \arctan \frac{x_{3} \arctan s}{1+\frac{1}{2} x_{3} \ln \left(s^{2}+1\right)}\right)^{2}\right\rangle\right]^{2}
\end{aligned}
$$




$$
\begin{aligned}
& \left.+\left[x_{1} \arctan \frac{x_{2} \arctan \frac{x_{3} \arctan s}{1+\frac{1}{2} x_{3} \ln \left(s^{2}+1\right)}}{1+\frac{1}{2} x_{2} \ln \left\langle\left[1+\frac{1}{2} x_{3} \ln \left(s^{2}+1\right)\right]^{2}+\left(x_{3} \arctan s\right)^{2}\right\rangle}\right]^{2}\right\} \\
\triangleq & \frac{1}{2} \ln \left[U_{3}^{2}\left(x_{1}, x_{2}, x_{3} ; s\right)+V_{3}^{2}\left(x_{1}, x_{2}, x_{3} ; s\right)\right],
\end{aligned}
$$

where $U_{1}\left(x_{1} ; s\right)$ and $V_{1}\left(x_{1} ; s\right)$ are defined by 2.10 and

$$
\begin{aligned}
U_{2}\left(x_{1}, x_{2} ; s\right) & =1+\frac{1}{2} x_{1} \ln \left[U_{1}^{2}\left(x_{2} ; s\right)+V_{1}^{2}\left(x_{2} ; s\right)\right], \\
V_{2}\left(x_{1}, x_{2} ; s\right) & =x_{1} \arctan \frac{V_{1}\left(x_{2} ; s\right)}{U_{1}\left(x_{2} ; s\right)}, \\
U_{3}\left(x_{1}, x_{2}, x_{3} ; s\right) & =1+\frac{1}{2} x_{1} \ln \left[U_{2}^{2}\left(x_{2}, x_{3} ; s\right)+V_{2}^{2}\left(x_{2}, x_{3} ; s\right)\right], \\
V_{3}\left(x_{1}, x_{2}, x_{3} ; s\right) & =x_{1} \arctan \frac{V_{2}\left(x_{2}, x_{3} ; s\right)}{U_{2}\left(x_{2}, x_{3} ; s\right)} .
\end{aligned}
$$

Assume that the recurrence relations 2.11 and 2.12 are valid for some $m \geq 3$. This means that

$$
\Re\left[H\left(s i ; \boldsymbol{x}_{m}\right)\right]=\frac{1}{2} \ln \left[U_{m}^{2}\left(x_{1}, \ldots, x_{m} ; s\right)+V_{m}^{2}\left(x_{1}, \ldots, x_{m} ; s\right)\right]
$$

and

for $m \geq 3$. Since

$$
\Im\left[H\left(s i ; \boldsymbol{x}_{m}\right)\right]=\arctan \frac{V_{m}\left(x_{1}, \ldots, x_{m} ; s\right)}{U_{m}\left(x_{1}, \ldots, x_{m} ; s\right)}
$$

$$
\begin{aligned}
H\left(s i ; \boldsymbol{x}_{m+1}\right) & =\ln \left[1+x_{1} H\left(s i ; x_{2}, \ldots, x_{m+1}\right)\right] \\
& =\ln \left(1+x_{1} \Re\left[H\left(s i ; x_{2}, \ldots, x_{m+1}\right)\right]+i x_{1} \Im\left[H\left(s i ; x_{2}, \ldots, x_{m+1}\right)\right]\right) \\
= & \frac{1}{2} \ln \left[\left(1+x_{1} \Re\left[H\left(s i ; x_{2}, \ldots, x_{m+1}\right)\right]\right)^{2}+\left(x_{1} \Im\left[H\left(s i ; x_{2}, \ldots, x_{m+1}\right)\right]\right)^{2}\right] \\
+ & i \arg \frac{x_{1} \Im\left[H\left(s i ; x_{2}, \ldots, x_{m+1}\right)\right]}{1+x_{1} \Re\left[H\left(s i ; x_{2}, \ldots, x_{m+1}\right)\right]}
\end{aligned}
$$

it follows that

$$
\begin{aligned}
\Re\left[H\left(s i ; \boldsymbol{x}_{m+1}\right)\right] & =\frac{1}{2} \ln \left[\left(x_{1} \arctan \frac{V_{m}\left(x_{2}, \ldots, x_{m+1} ; s\right)}{U_{m}\left(x_{2}, \ldots, x_{m+1} ; s\right)}\right)^{2}\right. \\
& \left.+\left(1+\frac{1}{2} x_{1} \ln \left[U_{m}^{2}\left(x_{2}, \ldots, x_{m+1} ; s\right)+V_{m}^{2}\left(x_{2}, \ldots, x_{m+1} ; s\right)\right]\right)^{2}\right]
\end{aligned}
$$

and

$$
\Im\left[H\left(s i ; \boldsymbol{x}_{m+1}\right)\right]=\arg \frac{x_{1} \arctan \frac{V_{m}\left(x_{2}, \ldots, x_{m+1} ; s\right)}{U_{m}\left(x_{2}, \ldots, x_{m+1} ; s\right)}}{1+\frac{1}{2} x_{1} \ln \left[U_{m}^{2}\left(x_{2}, \ldots, x_{m+1} ; s\right)+V_{m}^{2}\left(x_{2}, \ldots, x_{m+1} ; s\right)\right]} .
$$

These imply that

$$
\begin{gathered}
U_{m+1}\left(x_{1}, \ldots, x_{m+1} ; s\right)=1+\frac{1}{2} x_{1} \ln \left[U_{m}^{2}\left(x_{2}, \ldots, x_{m+1} ; s\right)+V_{m}^{2}\left(x_{2}, \ldots, x_{m+1} ; s\right)\right] \\
V_{m+1}\left(x_{1}, \ldots, x_{m+1} ; s\right)=x_{1} \arctan \frac{V_{m}\left(x_{2}, \ldots, x_{m+1} ; s\right)}{U_{m}\left(x_{2}, \ldots, x_{m+1} ; s\right)}
\end{gathered}
$$


INTEGRAL REPRESENTATIONS OF MULTIVARIATE LOGARITHMIC POLYNOMIALS 9

and

$$
\Im\left[H\left(s i ; \boldsymbol{x}_{m+1}\right)\right]=\arctan \frac{V_{m+1}\left(x_{1}, \ldots, x_{m+1} ; s\right)}{U_{m+1}\left(x_{1}, \ldots, x_{m+1} ; s\right)} .
$$

By mathematical induction, the formulas $(2.8)$ and $(2.9)$ and the recurrence relations 2.11 and 2.12 are valid. The proof of Theorem 2.3 is complete.

Theorem 2.4. For $m \in \mathbb{N}$ and $s, x_{1}, \ldots, x_{m}>0$, we have the integral formulas

$$
\begin{aligned}
\int_{0}^{\infty}\left[1-\cos \left(s_{m} s\right)\right] \int_{0}^{\infty} \cdots & \int_{0}^{\infty} \mathcal{Q}\left(\boldsymbol{s}_{m} ; \boldsymbol{x}_{m}\right) \mathrm{d} s_{0} \cdots \mathrm{d} s_{m-1} \mathrm{~d} s_{m} \\
& =\frac{1}{2} \ln \left[U_{m}^{2}\left(x_{1}, \ldots, x_{m} ; s\right)+V_{m}^{2}\left(x_{1}, \ldots, x_{m} ; s\right)\right]
\end{aligned}
$$

and

$$
\begin{aligned}
\int_{0}^{\infty} \sin \left(s_{m} s\right) \int_{0}^{\infty} \cdots \int_{0}^{\infty} \mathcal{Q}\left(\boldsymbol{s}_{m} ; \boldsymbol{x}_{m}\right) \mathrm{d} s_{0} \cdots & \mathrm{d} s_{m-1} \mathrm{~d} s_{m} \\
& =\arctan \frac{V_{m}\left(x_{1}, \ldots, x_{m} ; s\right)}{U_{m}\left(x_{1}, \ldots, x_{m} ; s\right)}
\end{aligned}
$$

where $\mathcal{Q}\left(\boldsymbol{s}_{m} ; \boldsymbol{x}_{m}\right), U_{m}\left(x_{1}, \ldots, x_{m} ; s\right)$, and $V_{m}\left(x_{1}, \ldots, x_{m} ; s\right)$ are defined by 2.2 , 2.10, 2.11, and 2.12 respectively.

Proof. By virtue of (2.1), we obtain

$$
\begin{aligned}
& H\left(s i ; \boldsymbol{x}_{m}\right)=\int_{0}^{\infty}\left(1-e^{-s_{m} s i}\right) \int_{0}^{\infty} \cdots \int_{0}^{\infty} \mathcal{Q}\left(\boldsymbol{s}_{m} ; \boldsymbol{x}_{m}\right) \mathrm{d} s_{0} \cdots \mathrm{d} s_{m-1} \mathrm{~d} s_{m} \\
= & \int_{0}^{\infty}\left[1-\cos \left(s_{m} s\right)+i \sin \left(s_{m} s\right)\right] \int_{0}^{\infty} \cdots \int_{0}^{\infty} \mathcal{Q}\left(\boldsymbol{s}_{m} ; \boldsymbol{x}_{m}\right) \mathrm{d} s_{0} \cdots \mathrm{d} s_{m-1} \mathrm{~d} s_{m}
\end{aligned}
$$

which means that

$$
\Re\left[H\left(s i ; \boldsymbol{x}_{m}\right)\right]=\int_{0}^{\infty}\left[1-\cos \left(s_{m} s\right)\right] \int_{0}^{\infty} \cdots \int_{0}^{\infty} \mathcal{Q}\left(\boldsymbol{s}_{m} ; \boldsymbol{x}_{m}\right) \mathrm{d} s_{0} \cdots \mathrm{d} s_{m-1} \mathrm{~d} s_{m}
$$

and

$$
\Im\left[H\left(s i ; \boldsymbol{x}_{m}\right)\right]=\int_{0}^{\infty} \sin \left(s_{m} s\right) \int_{0}^{\infty} \cdots \int_{0}^{\infty} \mathcal{Q}\left(\boldsymbol{s}_{m} ; \boldsymbol{x}_{m}\right) \mathrm{d} s_{0} \cdots \mathrm{d} s_{m-1} \mathrm{~d} s_{m} .
$$

Combining these with 2.8 and 2.9 yields 2.13 and 2.14 respectively. The proof of Theorem 2.4 is complete.

Remark 2.2. Taking $m=1$ in 2.13 and 2.14 can derive

$$
\begin{aligned}
\int_{0}^{\infty} \int_{0}^{\infty} \frac{v^{u x-1}}{\Gamma(u x+1)}[1-\cos (v s)] e^{-(u+v)} \mathrm{d} u \mathrm{~d} v & \\
= & \frac{1}{2 x} \ln \left\{\left[1+\frac{1}{2} x \ln \left(s^{2}+1\right)\right]^{2}+(x \arctan s)^{2}\right\}
\end{aligned}
$$

and

$$
\int_{0}^{\infty} \int_{0}^{\infty} \frac{v^{u x-1}}{\Gamma(u x+1)} \sin (v s) e^{-(u+v)} \mathrm{d} u \mathrm{~d} v=\frac{1}{x} \arctan \frac{x \arctan s}{1+\frac{1}{2} x \ln \left(s^{2}+1\right)}
$$

for $s, x>0$. 
Theorem 2.5. For $\Re(z) \geq 0$ and $x_{1}, \ldots, x_{m}>0$ with $m \in \mathbb{N}$, the integral representation

$$
H\left(z ; \boldsymbol{x}_{m}\right)=\frac{1}{\pi} \int_{0}^{\infty} \frac{z}{z^{2}+s^{2}} \ln \left[U_{m}^{2}\left(x_{1}, \ldots, x_{m} ; s\right)+V_{m}^{2}\left(x_{1}, \ldots, x_{m} ; s\right)\right] \mathrm{d} z
$$

is pointwisely convergent but not uniformly convergent, where $U_{m}\left(x_{1}, \ldots, x_{m} ; s\right)$ and $V_{m}\left(x_{1}, \ldots, x_{m} ; s\right)$ are defined by 2.10, , 2.11), and $(2.12)$ respectively. Consequently, the integrals in (1.6) and (1.7) are pointwisely convergent but not uniformly convergent.

Proof. A simple computation gives

$$
\lim _{t \rightarrow 0} H\left(t ; \boldsymbol{x}_{m}\right)=\lim _{t \rightarrow \infty} \frac{H\left(t ; \boldsymbol{x}_{m}\right)}{t}=0 .
$$

Accordingly, when $t \geq 0$ and $x_{1} \ldots, x_{m}>0$, by (1.6) or 1.7), it follows that

$$
H\left(t ; \boldsymbol{x}_{m}\right)=\frac{2}{\pi} \int_{0}^{\infty} \frac{t}{t^{2}+s^{2}} \Re\left[G\left(s i ; \boldsymbol{x}_{m}\right)\right] \mathrm{d} s .
$$

If the integrals in 1.6 and 1.7 are uniformly convergent, then differentiating on both sides of the above integral representation for $H\left(t ; \boldsymbol{x}_{m}\right)$ results in

$$
\frac{\partial^{k} H\left(t ; \boldsymbol{x}_{m}\right)}{\partial t^{k}}=\frac{1}{\pi} \int_{0}^{\infty} \frac{\partial^{k}}{\partial t^{k}}\left(\frac{2 t}{t^{2}+s^{2}}\right) \Re\left[G\left(s i ; \boldsymbol{x}_{m}\right)\right] \mathrm{d} s
$$

for $t \geq 0$ and $k \in \mathbb{N}$, where

$$
\begin{aligned}
\frac{\partial^{k}}{\partial t^{k}}\left(\frac{2 t}{t^{2}+s^{2}}\right) & =\frac{\partial^{k+1} \ln \left(t^{2}+s^{2}\right)}{\partial t^{k+1}}=\sum_{\ell=1}^{k+1} \frac{\mathrm{d}^{\ell} \ln \mu}{\mathrm{d} \mu^{\ell}} \mathrm{B}_{k+1, \ell}(2 t, 2,0, \ldots, 0) \\
& =\sum_{\ell=1}^{k+1} \frac{(-1)^{\ell-1}(\ell-1) !}{\mu^{\ell}} 2^{\ell} \mathrm{B}_{k+1, \ell}(t, 1,0, \ldots, 0) \\
& =\sum_{\ell=1}^{k+1} \frac{(-1)^{\ell-1}(\ell-1) !}{\left(t^{2}+s^{2}\right)^{\ell}} 2^{\ell} \frac{(k-\ell+1) !}{2^{k-\ell+1}}\left(\begin{array}{c}
k+1 \\
\ell
\end{array}\right)\left(\begin{array}{c}
\ell \\
k-\ell+1
\end{array}\right) t^{2 \ell-k-1} \\
& =(k+1) ! \sum_{\ell=1}^{k+1} \frac{(-1)^{\ell-1}}{\left(t^{2}+s^{2}\right)^{\ell}} \frac{1}{\ell}\left(\begin{array}{c}
\ell \\
k-\ell+1
\end{array}\right)(2 t)^{2 \ell-k-1} \\
& \rightarrow\left\{\begin{array}{cc}
0, & k \text { is even } \\
(-1)^{(k-1) / 2} \frac{2(k !)}{s^{k+1}}, & k \text { is odd }
\end{array}\right.
\end{aligned}
$$

as $t \rightarrow 0$ for $k \in \mathbb{N}$, where $\mu=\mu(t)=t^{2}+s^{2}$ and the formula

$$
\mathrm{B}_{n, k}(x, 1,0, \ldots, 0)=\frac{(n-k) !}{2^{n-k}}\left(\begin{array}{l}
n \\
k
\end{array}\right)\left(\begin{array}{c}
k \\
n-k
\end{array}\right) x^{2 k-n}, \quad 0 \leq k \leq n
$$

in [11, Theorem 5.1] were used. Taking $t \rightarrow 0$ in (2.16) and making use of (1.9) in Definition 1.1 conclude

$$
\frac{\partial^{k} H\left(t ; \boldsymbol{x}_{m}\right)}{\partial t^{k}}=L_{m, k}\left(\boldsymbol{x}_{m}\right)= \begin{cases}0, & k \text { is even } \\ (-1)^{(k-1) / 2} \frac{2(k !)}{\pi} \int_{0}^{\infty} \frac{\Re\left[G\left(s i ; \boldsymbol{x}_{m}\right)\right]}{s^{k+1}} \mathrm{~d} s, & k \text { is odd }\end{cases}
$$

for $k \in \mathbb{N}$. This leads to a contradiction. The proof of Theorem 2.5 is complete. 
Remark 2.3. Theorem 2.5 demonstrates that we can not derive an integral representation for multivariate logarithmic polynomials $L_{m, n}\left(\boldsymbol{x}_{m}\right)$ from the integral representation 2.15 .

\section{REFERENCES}

[1] M. Abramowitz and I.A. Stegun (Eds), Handbook of Mathematical Functions with Formulas, Graphs, and Mathematical Tables, National Bureau of Standards, Applied Mathematics Series 55, 10th printing, Washington, 1972.

[2] C. Berg, A Pick function related to an inequality for the entropy function, J. Inequal. Pure Appl. Math. 2 (2001), no. 2, Art. 26; Available online at http://www.emis.de/journals/ JIPAM/article142.html.

[3] C. Berg and H. L. Pedersen, A one-parameter family of Pick functions defined by the Gamma function and related to the volume of the unit ball in $n$-space, Proc. Amer. Math. Soc. 139 (2011), no. 6, 2121-2132; Available online at https://doi.org/10.1090/ S0002-9939-2010-10636-6

[4] C.-P. Chen, F. Qi, and H. M. Srivastava, Some properties of functions related to the gamma and psi functions, Integral Transforms Spec. Funct. 21 (2010), no. 2, 153-164; Available online at https://doi.org/10.1080/10652460903064216

[5] S. Fourati and W. Jedidi, Some remarks on the class of Bernstein functions and some subclasses, available online at https://hal.archives-ouvertes.fr/hal-00649018

[6] S. Fourati and W. Jedidi, Some remarks on the class of Bernstein functions and some subclasses, ResearchGate Research (2016), available online at https://doi.org/10.13140/RG. 2.1 .3431 .0169

[7] S. Fourati and W. Jedidi, Some remarks on the class of Bernstein functions and some subclasses, ResearchGate Article (2011), available online at https://www.researchgate.net/ publication/281599574.

[8] I. S. Gradshteyn and I. M. Ryzhik, Table of Integrals, Series, and Products, Translated from the Russian, Translation edited and with a preface by Daniel Zwillinger and Victor Moll, Eighth edition, Revised from the seventh edition, Elsevier/Academic Press, Amsterdam, 2015; Available online at https://doi.org/10.1016/B978-0-12-384933-5.00013-8

[9] D. S. Mitrinović, J. E. Pečarić, and A. M. Fink, Classical and New Inequalities in Analysis, Kluwer Academic Publishers, 1993; Available online at https://doi.org/10.1007/ 978-94-017-1043-5

[10] F. Qi, On multi-order logarithmic polynomials and their explicit formulas, recurrence relations, and inequalities, Preprints 2017, 2017090034, 11 pages; Available online at https: //doi.org/10.20944/preprints201709.0034.v1

[11] F. Qi and B.-N. Guo, Explicit formulas for special values of the Bell polynomials of the second kind and for the Euler numbers and polynomials, Mediterr. J. Math. 14 (2017), no. 3, Article 140, 14 pages; Available online at https://doi.org/10.1007/s00009-017-0939-1

[12] F. Qi and X.-J. Zhang, An integral representation, some inequalities, and complete monotonicity of the Bernoulli numbers of the second kind, Bull. Korean Math. Soc. 52 (2015), no. 3, 987-998; Available online at https://doi.org/10.4134/BKMS.2015.52.3.987

[13] R. L. Schilling, R. Song, and Z. Vondraček, Bernstein Functions-Theory and Applications, 2nd ed., de Gruyter Studies in Mathematics 37, Walter de Gruyter, Berlin, Germany, 2012; Available online at https://doi.org/10.1515/9783110269338

[14] D. V. Widder, The Laplace Transform, Princeton University Press, Princeton, 1946. 